\title{
Floating Vitreous Cyst: Two Clinical Cases
}

\author{
Alenka Lavric Mojca Urbancic \\ Eye Hospital, University Medical Centre Ljubljana, Ljubljana, Slovenia
}

\section{Key Words}

Vitreous cyst · Floater · Retinoschisis · Myopia

\begin{abstract}
Purpose: To report two cases of solitary unilateral vitreous cyst. Methods: A complete ocular examination, fundus photography, B-scan ultrasound and spectral-domain optical coherence tomography were performed in both patients. Results: The first patient (a 39-year-old man) presented with transient blurred vision in the right eye. The second patient (a 78-year-old man) reported transient blurred vision in the right eye when changing head position. He was referred to the Eye Hospital because of vitreomacular traction in the other eye. After examination, a diagnosis of vitreous cyst was made in both cases. Conclusions: Vitreous cysts are rare clinical findings. They can occur in normal eyes or in eyes with certain ocular pathologies. When a cyst floats into the visual axis area, it can disturb visual function; therefore, patients usually report transient blurring of vision. A prompt clinical examination is necessary for differentiating this rare condition.

(C) 2013 S. Karger AG, Basel
\end{abstract}

\section{Introduction}

Most authors classify vitreous cysts into congenital or acquired. The former are believed to be remnants of the hyaloid vascular system such as Bergmeister's papilla and Mittendorf's dot [1]. They are grey or pearly, mostly nonpigmented and usually located in the posterior vitreous and fixed to the surface of the optic disc. On the contrary, pigmented cysts arise from the iris or ciliary body pigment epithelium [2]. If they get dislodged, they can migrate into the anterior chamber, in the vitreous or can be fixed in the anterior chamber angle. These cysts are usually oval and brown, and may have a different amount of pigment. Congenital cysts are stable, remain unchanged for a long period of time, and seldom cause visual symptoms. They are not associated with concomitant or previous eye pathology. 
Lavric et al.: Floating Vitreous Cyst: Two Clinical Cases

Acquired cysts may be due to ocular trauma, inflammatory diseases such as toxoplasmosis [3] or intermediate uveitis [4]. They can also be associated with degenerative diseases of the retina and choroid, like high myopia with uveal coloboma [5] and retinal detachment surgeries [6].

\section{Case Descriptions}

Case 1

A 39-year-old, highly myopic man with a 2-day history of a dark object floating in his right eye was examined in our clinic. The patient had no history of ocular trauma or intraocular inflammation. Best-corrected visual acuity was 0.4 in the right eye and 0.9 in the left eye. The anterior segments were normal in both eyes. Dilated fundus examination revealed slightly tilted optic discs with peripapillary atrophy due to high myopia and small flame-shaped hemorrhage on the temporal side of the right optic disc (fig. 1a). Posterior vitreous detachment (PVD) and an oval, opaque, and pedunculated cyst was seen in the vitreous cavity of the right eye (fig. 1b). The free-floating cyst was located in the inferior vitreous when the eye was in primary position and obscured the underlying retina. Fundus images were taken with a fundus camera and a spectral-domain optical coherence tomography (SD-OCT) (Heidelberg Engineering, Heidelberg, Germany) was performed (fig. 1d). Bscan ultrasound of the right eye demonstrated intravitreal opacities and confirmed the presence of a hyperechogenic cyst with no internal reflectivity (fig. 1c). The diameter of the cyst was $3.1 \mathrm{~mm}$. Blood serology tests were negative for Toxoplasma gondii, Toxocara canis, cysticercosis and Echinococcus. Observation of the cyst was advised.

Case 2

A 78-year-old man was referred to the Eye Hospital because of vitreomacular traction in the left eye. He reported a slow visual deterioration in his left eye over the last 2 years, but had no problems with the right eye. Only when specifically asked, he reported transient blurring of vision in his right eye when changing head position. His medical history was unremarkable. The patient's best-corrected visual acuity was 0.5 in the right eye and 0.3 in the left eye. Slit-lamp examination showed nuclear cataract in both eyes, anterior segments were otherwise normal. Fundus examination of the right eye showed a big, round, semitranslucent, and partially pigmented vitreous cyst just below the inferior temporal vascular arcades (fig. 2a, b). The cyst appeared empty. During eye movements, the cyst was freely floating in the vitreous cavity. Inferior temporal retinoschisis was also noted in this eye, but there were no other changes of the fundus. Fundus examination of the left eye revealed significant vitreomacular traction. B-scan ultrasonography of the right eye showed partial PVD and a cystic, well-demarcated formation not fixed to any other ocular structures, with a diameter of $6 \mathrm{~mm}$ (fig. 2c). The cyst was evaluated also by SD-OCT. Images confirmed a thin, well-defined cyst wall with a hyporeflective lumen. Inside the cyst, small hyperreflective spots were present (fig. 2d, e). Blood serology tests were negative for Toxoplasma gondii, Toxocara canis, cysticercosis and Echinococcus. Observation was recommended as in the first case. 
Lavric et al.: Floating Vitreous Cyst: Two Clinical Cases

\section{Discussion}

Vitreous cysts represent an uncommon condition and are usually incidental clinical findings. A few possible etiologies have been proposed. The theory of a residual hyaloid system as a cyst's origin is supported by histopathological findings of Nork and Millecchia [7], which suggest that the cyst is a choristoma of the primary hyaloid system. Orellana et al. [8] studied aspirated pigmented cysts by light and electron microscope and observed that the pigmented layer of cuboidal cells contains mature melanosomes and some immature melanosomes, supporting the theory that the cyst originates from the pigment epithelium.

Both of our patients had only mild visual symptoms, therefore, surgical removal and histopathological studies of the cyst were not performed, and we can only speculate on the exact origin of the cyst. Both patients had no history of ocular trauma or inflammation and had a unilateral, solitary cyst. The first patient had an oval, grey cyst with a stalk-like extension that may be associated with the hyaloid system. The patient's sudden symptoms of a dark floater could be caused by PVD, which might produce dislocation of the cyst from the attachment to the optic disc. This assumption is supported by the presence of peripapillary hemorrhage. It is known that vitreopapillary traction can traumatize disc vessels, causing hemorrhage in or around the disc. Eyes with a tilted disc architecture associated with myopia may have an unusual vitreous attachment. In these eyes, the peripapillary intraretinal hemorrhage most commonly occurs because of vitreous separation [9]. In the second patient, the appearance of the cyst leads to the assumption that this round-shaped cyst with pigment clumps in the wall and no evident residues of hyaloid artery or primary vitreous originates from the iris or ciliary body pigment epithelium. The presence of peripheral retinoschisis may also suggest the ciliary body origin of the vitreous cyst. It is known that a pars plana cyst can occur in retinoschisis. Mannino et al. [10] showed a topographical and morphological relation of pars plana cysts with peripheral degenerative retinoschisis. We assume that an isolated pars plana cyst was somehow dislodged from its primary position. The possibility of a vitreous reaction to the underlying retinal degeneration must also be considered as a mechanism of cyst formation in both cases (degenerative myopia with PVD in the first patient and degenerative retinoschisis with partial PVD in the second patient).

Free-floating vitreal cysts are usually of little clinical importance, but it is important to differentiate them from potentially serious conditions. Pigmented cysts can be mistaken for pigmented tumors like malignant melanoma [5]. Grey, opaque cysts may resemble parasitic cysts like in cysticercosis [11]. Besides clinical examination, serological tests for intraocular infection, ultrasound or OCT detection of scolex inside the cyst [12] may help to make the correct diagnosis.

Although many cases of vitreous cyst have been described in the literature, to our knowledge only one documented the OCT features of vitreous cysts [13]. The previously described cases most commonly included color images and ultrasound evidence. OCT may represent an additional diagnostic tool for cyst wall and content documentation, especially when the cyst is located near the posterior pole making the OCT scanning possible.

No treatment has been performed in our cases, but regular follow-up was recommended. Argon laser [14], neodymium:YAG laser treatment [15], and surgical removal of the cyst have been described in the literature [8] as possible treatment options in symptomatic cysts.

\section{Disclosure Statement}

The authors have no conflicts of interest to disclose. 


\begin{tabular}{l|l}
\hline Case Rep Ophthalmol 2013;4:243-247 \\
\hline DOI: $10.1159 / 000356569$ & $\begin{array}{l}\text { C 2013 S. Karger AG, Basel } \\
\text { www.karger.com/cop }\end{array}$ \\
\hline
\end{tabular}

Lavric et al.: Floating Vitreous Cyst: Two Clinical Cases

\section{References}

1 Bullock JD: Developmental vitreous cysts. Arch Ophthal 1974;91:83-84.

-2 Lisch W, Rochels R: Pathogenesis of congenital vitreous cysts (in German). Klin Monbl Augenheilkd 1989;195:375-378.

-3 Pannarale C: On a case of preretinal mobile cysts in a subject affected by congenital toxoplasmosis (in Italian). G Ital Oftalmol 1964;17:306-317.

-4 Tranos PG, Ferrante P, Pavesio C: Posterior vitreous cyst and intermediate uveitis. Eye 2010;24:1115-1116.

-5 Tuncer S, Bayramoglu S: Pigmented free-floating vitreous cyst in a patient with high myopia and uveal coloboma simulating choroidal melanoma. Ophthalmic Surg Lasers Imaging 2011;42(Online):e49-e52.

-6 Asiyo-Vogel MN, el-Hifnawi el-S, Laqua H: Ultrastructural features of a solitary vitreous cyst. Retina 1996;16:250-254.

7 Nork TM, Millecchia LL: Treatment and histopathology of a congenital vitreous cyst. Ophthalmology 1998;105:825-830.

8 Orellana J, O'Malley RE, McPherson AR, Font RL: Pigmented free-floating vitreous cysts in two young adults. Electron microscopic observations. Ophthalmology 1985;92:297-302.

-9 Katz B, Hoyt WF: Intrapapillary and peripapillary hemorrhage in young patients with incomplete posterior vitreous detachment. Signs of vitreopapillary traction. Ophthalmology 1995;102:349-354.

10 Mannino G, Malagola R, Abdolrahimzadeh S, Villani GM, Recupero SM: Ultrasound biomicroscopy of the peripheral retina and the ciliary body in degenerative retinoschisis associated with pars plana cysts. Br J Ophthalmol 2001;85:976-982.

11 Wood TR, Binder PS: Intravitreal and intracameral cysticercosis. Ann Ophthalmol 1979;11:1033-1036.

12 Sinha S, Takkar B, Venkatesh P, Khanduja S: High-resolution Fourier-domain optical coherence tomography findings in subretinal cysticercosis. Retina 2012;32:643-644.

$\$ 13$ Basdekidou C, Wolfensberger TJ: Six-year dynamic growth pattern of two concentric congenital vitreous cysts. Eye 2010;24:1301-1303.

14 Awan KJ: Biomicroscopy and argon laser photocystotomy of free-floating vitreous cysts. Ophthalmology 1985;92:1710-1711.

15 Ruby AJ, Jampol LM: Nd:YAG treatment of a posterior vitreous cyst. Am J Ophthalmol 1990;110:428-429.

16 Sadda SR, Keane P: Pitfalls to consider when using Spectral domain OCT. Retina Today 2009;3:38-41.
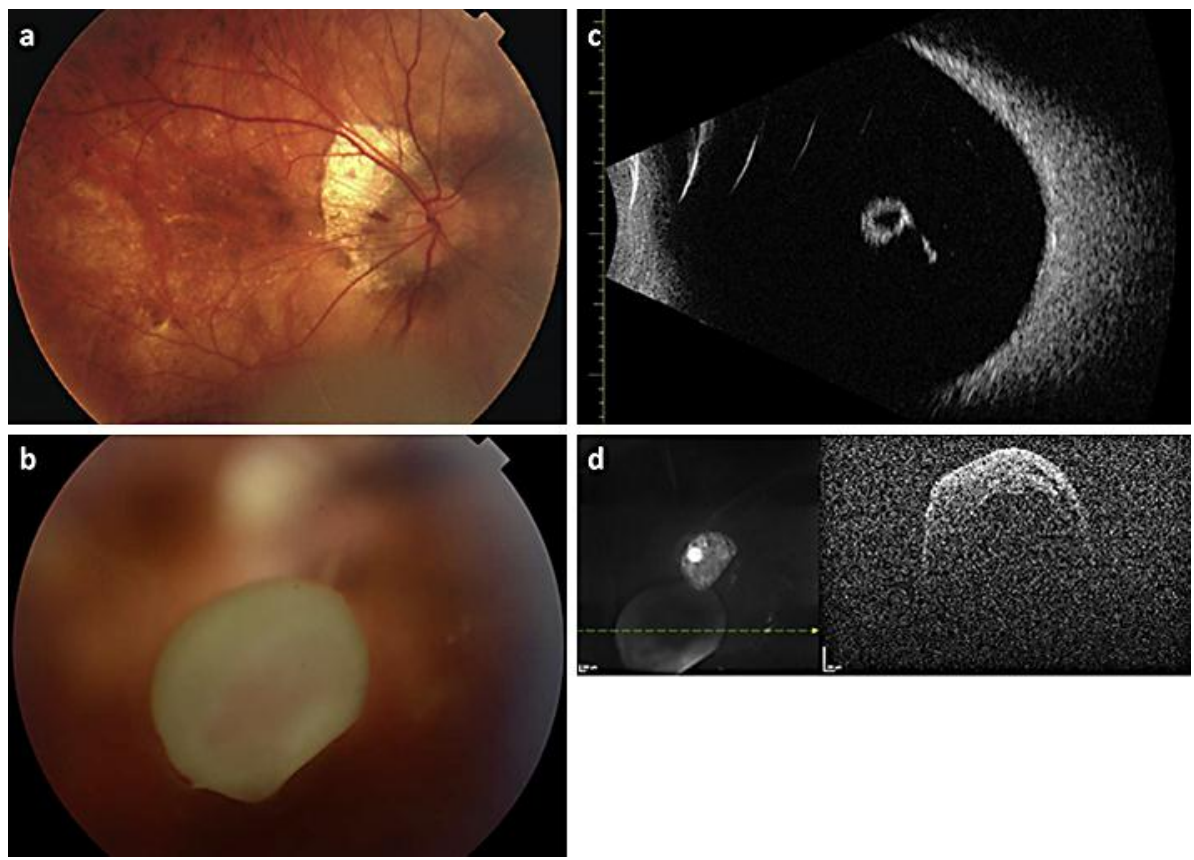

Fig. 1. Case 1. a Color fundus image of the right eye with peripapillary atrophy due to degenerative myopia and with a small intraretinal flame-shaped hemorrhage on the temporal part of the optic disc. $\mathbf{b}$ Grey, pedunculated vitreous cyst without pigment on its wall. c B-scan ultrasound (20 MHz; Aviso S; Quantel Medical, France) showing a free-floating cyst with a stalk-like extension. $\mathbf{d}$ SD-OCT showing a cyst with thick, irregular walls. 
Lavric et al.: Floating Vitreous Cyst: Two Clinical Cases
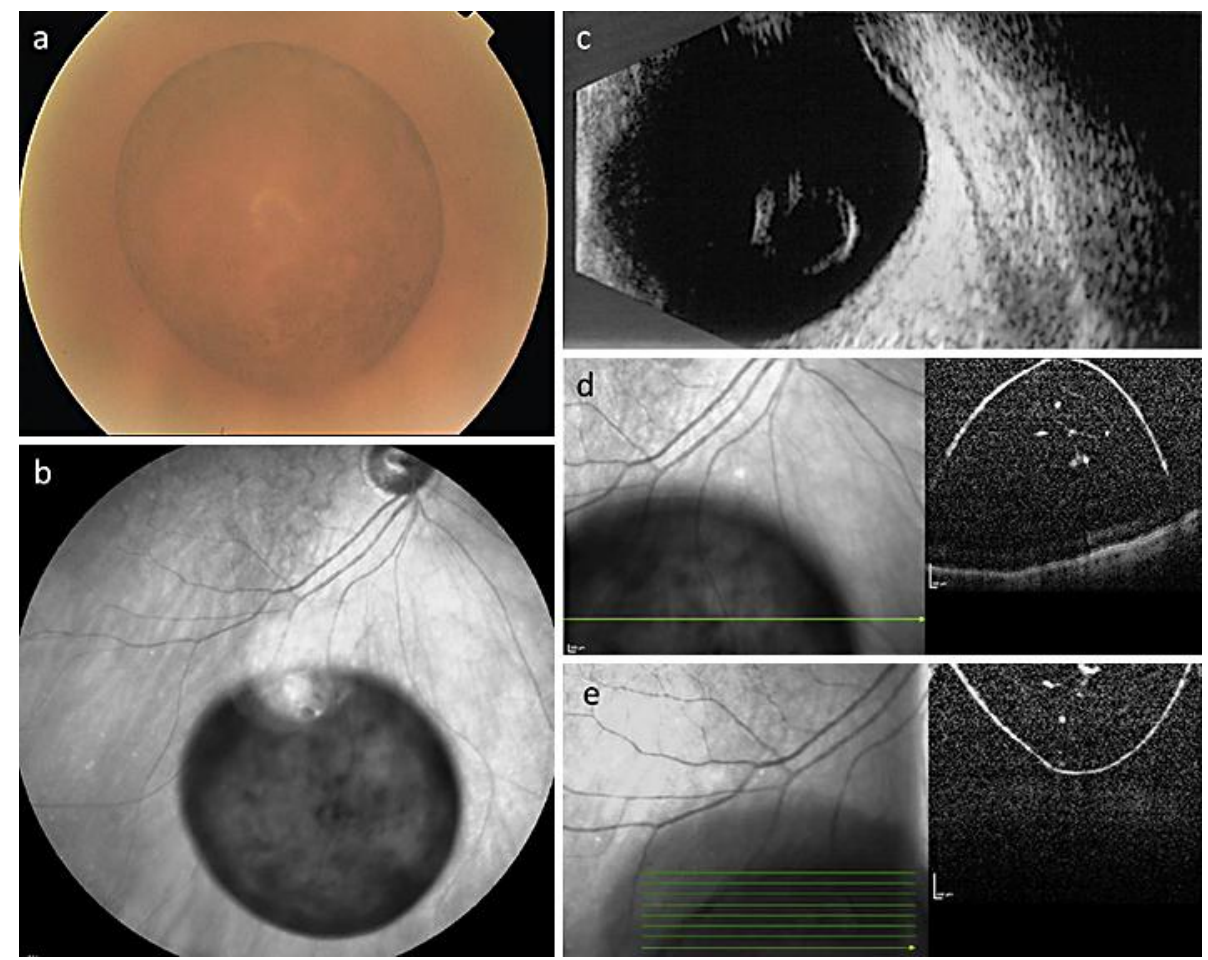

Fig. 2. Case 2. a A free-floating, partially pigmented cyst in the lower vitreous cavity is shown. $\mathbf{b}$ Infra-red image of the cyst with pigment clumping. c B-scan ultrasound (10 MHz; Cine-Scan S; Quantel Medical) of the cyst with reflective walls, clear contents and inferior temporal retinoschisis. d, e SD-OCT of the cyst with thin, smooth, and hyperreflective walls. Hyperreflective spots are seen inside the hyporeflective cyst which possibly correspond to pigment epithelial cells. The mirror image of a vitreous cyst is present in the first SD-OCT scan (d) due to a conjugate image artefact [16]. 